\title{
Study on the Application of Framework Expression in Knowledge Engineering Education Technique
}

\author{
Yafeng Zhang ${ }^{1, \text { a }}$, Yue Zhao ${ }^{2, \mathrm{~b}}$ and Xi Ouyang ${ }^{3, \mathrm{c}}$ \\ ${ }^{1}$ Dept. of Equipment Support, Bengbu Automobile NCO Academy, Bengbu 233011, China \\ ${ }^{2}$ Foreign Studies College, Northeastern University, Shengyang 110189, China \\ ${ }^{3}$ Dept. of Drive Training Service, Bengbu Automobile NCO Academy, Bengbu 233011, China \\ awillnudt@sina.com, b495822196@qq.com, '1013506189@qq.com
}

Keywords: Knowledge engineering technique (KEET); frame expression; structured knowledge; systematized knowledge.

\begin{abstract}
For construction of structured knowledge and systematized knowledge, this paper proposes the application of framework expression in knowledge Engineering Education Technique. Concept, key ideas, Inspiration and practice of framework expression in Knowledge engineering technique are discussed in this paper, which may play a positive role in the application of this method. The Knowledge engineering technique focuses on the process of problem-solving design, and the framework expression pays attention to the structured and systematized knowledge. The combination of the two may promote the quality of education.
\end{abstract}

\section{Introduction}

The cognitive psychology believes that only well-organized knowledge can be activated under certain stimulus and further extracted for application [1]. To help students construct structured knowledge and systematized knowledge is an important research subject in education [2-4]. Is it possible to help student achieve the construction of structured knowledge and systematized knowledge by using Knowledge Engineering Education Technique [5]. Some knowledge expression of computer gives us some enlightenment. Because of the diversity of knowledge expression, the design of education task can be very flexible and multiple in Knowledge engineering technique. For construction of structured knowledge and systematized knowledge, this paper proposes the application of framework expression in Knowledge engineering technique. Concept, key ideas, Inspiration and practice of framework expression in Knowledge engineering technique are discussed to explain the significance and rationality of this method. Unless otherwise stated, the frame expression refers to the frame expression in Knowledge engineering technique in the following discussion.

\section{Concept of framework expression in Knowledge engineering technique}

\subsection{Frame expressions in artificial intelligence}

M. Minsky proposed the frame expression theory in 1974. The frame theory states that people's understanding of all kinds of things in the real world is based on structures like frames stored in the brain. When people encounter new situations, such a structure will be selected from the memory and some of the structure details will be changed according to the actual need to adapt to the reality [6, 7]. It can be said that the frame expression is a data structure to express specific information. The frame expression supported by the theory was firstly used as knowledge expression of visual perception, natural language dialogue and other issues. The frame expression has been taken as a common data structure to express knowledge objects.

\subsection{Concept of frame expressions in Knowledge engineering technique}

The core idea of Knowledge engineering technique is to simulate computer to accumulate and express knowledge. It has to comply with the law of Knowledge engineering technique when we apply frame expression in Knowledge engineering technique. On the surface, frame expression of 
knowledge focuses on knowledge expression. But in fact, it is also inextricably linked with knowledge selection. Knowledge engineering technique states that the experience to achieve the purpose of solving problems allows us to building knowledge more effectively. Frame Theory reflects empiricism in a certain degree. Naturally, the experience to carry out frame expression enables us to accomplish other frame expression tasks more intelligently. Thus, the frame expression can be applied in Knowledge engineering technique. The frame expression of knowledge in Knowledge engineering technique refers to the frame structure where part or all of the educational tasks are expressed as both executable and instructive by simulating frame expression of computer. Frame expression brings together relevant characteristics of each educational task (including knowledge unit and knowledge set). It highly simulates the multi-faceted and multi-layered structure of the human brain in storing knowledge. The emphasis on the frame expression in Knowledge engineering technique is the frame expression of the events in educational tasks, which are obviously different from the emphasis on the structured knowledge in structured subject.

\section{Key ideas of framework expression}

The frame expression is a structured knowledge expression in Knowledge engineering technique. If an educational task uses the framework expression, the basic educational events represented by some of its terminals may become part of other educational tasks. In this way, these terminals can be shared. A terminal itself may contain a lot of educational information. Therefore, the terminal can be divided into smaller ones that have their own content and contain less information. Although one terminal of an educational frame expression may be shared by other educational tasks, there exist a certain constraint conditions for the selection and share of the terminal. If an educational task is now seen as a network full of educational information nodes, obviously this network will not be as monotonous as fishing net. This is because the frame expression of educational task is characterized by a multi-layered structure. We need to find a frame or a terminal in the basic events of the same level in educational tasks. Conversely, the terminal fillers of one educational task will be other terminals instead of frames. Consisting of a plurality of frame educational events, the basic structure of its frame system is formed by horizontal or vertical links between various educational events framework. The frame expressions of educational tasks that can be transplanted on the same level have horizontal linkages. If an educational task inherits the properties of another educational task and can be supplemented and modified, then the frame expressions of the two have vertical relationships.

\section{Inspiration of framework expression}

\subsection{Inspiration of framework expression for structured knowledge}

Structured knowledge means that knowledge is divided into different sections or classified into some larger areas, organized in the mind to form the knowledge chunks and further turned into a good knowledge structure [8]. When some specific educational tasks are expressed by frames, each frame or terminal may contain one or more educational information. Some of them tell how to use the educational knowledge, others are to forecast what might happen next in the educational task, and still others offer the countermeasures if the forecast fails. Such a frame expression is more conducive for structuring knowledge in educational tasks. It can also express the internal structure relationship of knowledge and the special relationship between knowledge. Moreover, the frame expression allows for the embedment of frame and terminal. As a result, it can express the educational tasks in a multifaceted and multi-attribute way. For example, the causal events in educational tasks can be expressed by frame expression. At the same time, the Embeddable frame and terminal are conducive for the inheritance and supplement of knowledge. It has great significance for the establishment and improvement of structured knowledge.

\subsection{Inspiration of framework expression for systematized knowledge}

A systematized body of knowledge means that knowledge can be organized according to the intrinsic link among each domain. Its feasibility and mobility facilitate the integration of new 
emerging knowledge with the system. For Knowledge engineering technique, the accumulation of underlying knowledge and the top-level design of educational tasks are not contradictory. Although structured knowledge is inseparable from the underlying knowledge structure, the systematized knowledge can be extensively used for educational tasks. If the multiple frames in educational tasks are seen as a network between educational information nodes and interrelations, an information retrieval network will help form the frame system by connecting each frame. Frame system will include many sub-frames. The top of one frame system is generally fixed. The educational events represented by it are almost always a true proposition in the hypothetical situation. Frame expression enables the corresponding educational task systems to have these features: the unresolved problems in educational tasks are studied at the system level and the overall system is designed based on the basic concepts, basic principles, basic structure and basic design methods. The learning process of educatees is how the low level of frame supports the presence of high-level framework while the latter contains and enriches the former. The framework represents the reticulate information structure of the general concepts stored in the memory. It has certain similarities in interdependence and interlocking among different thinking models. The framework can be interpreted as a group of interacting knowledge structure or the basic components of cognitive abilities stored in long-term memory by hierarchical levels. According to the learning theory given by cognitive psychology school, if the frame of the educational task is properly designed, educatees will gain their experience by accepting the training given by the frame, enhance their own ability to grasp the overall situation, and consciously form the habit to build knowledge system.

Educational Aims

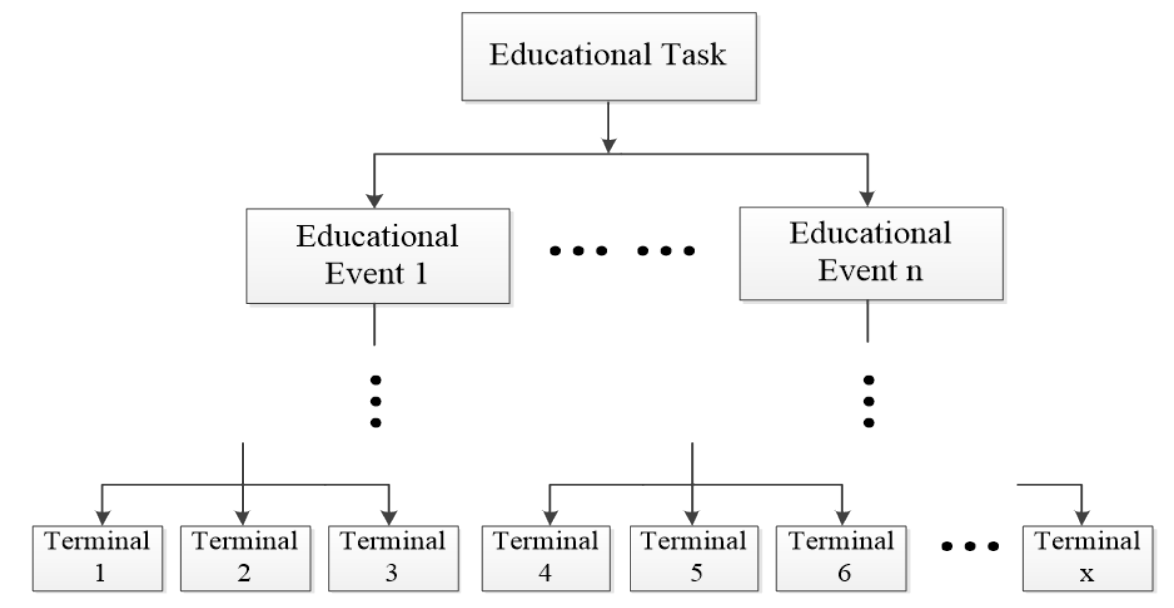

m Level Educational Objective

Fig. 1 The schematic of education frame longitudinal design

\section{Practice of framework expression}

Knowledge engineering technique practice principles allow the frame to be embedded into the design of educational task, or to design educational tasks independently. Before designing the frame of educational task, the educational tasks needs to be simplified and decomposed into some basic educational events. This was done for two reasons: firstly, the capacity of the frame and terminal base should not be infinite; secondly, a simplified educational task is easier to be expressed scientifically. The simplification and decomposition principle is that the collection of basic educational events must not only be small enough so as to be treated expediently, but also large enough to represent the main content of the educational tasks. For the practice of frame expression in Knowledge engineering technique, we need to analyze the educational tasks to recognize the knowledge chunk and network of the educational task and get a clear understanding of the knowledge level and knowledge system. On this basis, we can simplify the educational tasks and design its framework. By longitudinal design of framework, the target system, which is upgraded in progressive layers, can be established. The educational objectives at a lower level lay the foundation for those at a higher level. For the accumulation of knowledge and the cultivation of ability, the low-level educational objectives pave the way for the high-level educational objectives. All the objectives converge to the overall education 
objective. This design ensures that all the education links are expanded around the central educational objective. As a result, it's easy to get the whole picture while laying emphasis on the education task. The realization of the education objective at a certain level depends on the education tasks at the same level. On the basis of the longitudinal design of the framework, education events in every level can be determined by the horizontal design of framework in order to achieve the educational objective. The breadth of knowledge in every level should be reasonably grasped, which can meet the needs of the completion of the educational objective and will not affect the quality and progress of educational task because of redundancy. As is shown in Fig. 2, educational events in a certain level are not only supported by educational events in the lower level or even the terminal. Construction of knowledge system in the framework should accord with the laws of educational cognition and follow basic principles which work from simple to complex, from easy to difficult, from theory to practice. The practice should direct educatees to the principles and laws of frame expression in Knowledge engineering technique, and further understand the inner relationship and interaction between the frames. In this way, when facing the bottom terminal, educatees would refine knowledge systematically and microscopically, dig out the obscure knowledge to enrich knowledge base, connect bits of knowledge to weave a relatively complete knowledge network; When facing the top-level frame, it would help the educatees form a holistic understanding about the educational tasks, and further cultivate the ability of a comprehensive analysis.

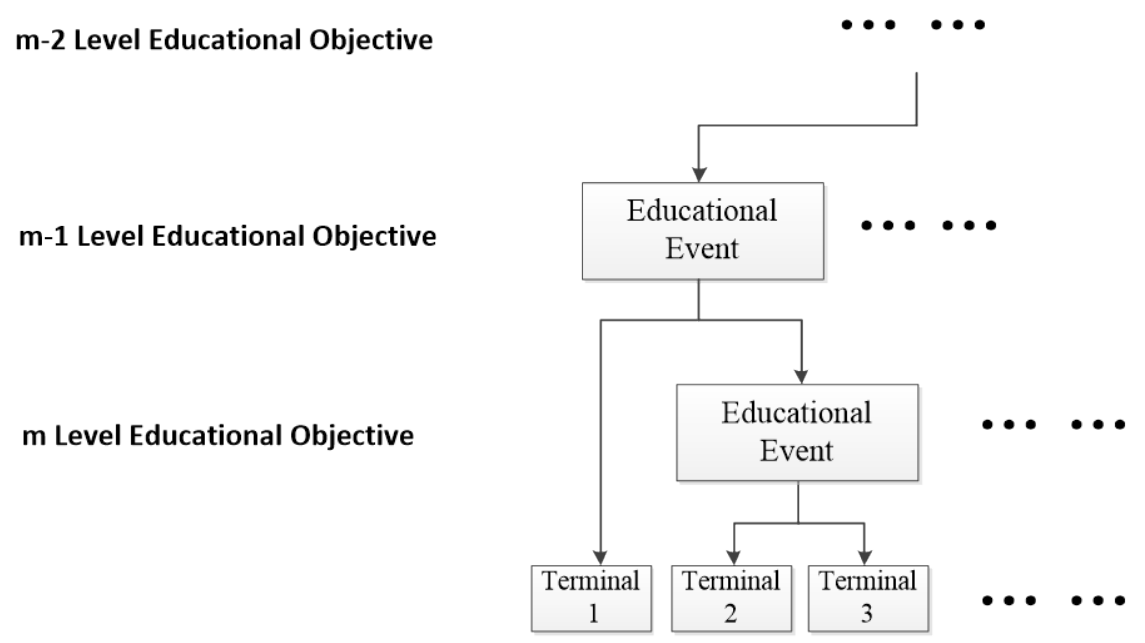

Fig. 2 The schematic of education frame horizontal design

\section{Summary}

For construction of structured knowledge and systematized knowledge, this paper proposes the application of framework expression in Knowledge engineering technique. Concepts, key ideas, Inspiration and practice of framework expression in Knowledge engineering technique are discussed to explain the significance and rationality of this method. Not only does the application of frame expression in Knowledge engineering technique focus on the process of problem-solving design, but it also pays attention to the structured and systematized knowledge. It may play a positive role in further promoting the quality of education.

\section{References}

[1]. B. R. Hergenhahn, M. H. Olson, Introduction to the Theories of Learning, Now York, Prentice Hall of Pearson Education, 2005.

[2]. H. X. Hong, L. H. Lv, "Gaps in Knowledge Structure of Chinese Students and the Alterative Approach (in Chinese),” Research in Educational Development, vol. 21, pp. 13-17, 2014. 
[3]. Y. F. Feng, G. X. Zhang,G. Rong, "Research-oriented experimental teaching based on systematic reconstruction of knowledge (in Chinese)," Experimental Technology and Management, vol. 29, pp. 154-156, Oct. 2012

[4]. H. C. Zhao, G. Liu, M. Ma, "A Review of Researches on Knowledge Construction under the Background of Information in China (in Chinese)," Journal of distance education, vol. 5, pp. 47-57, May 2014.

[5]. Y. F. Zhang, M. W. Zhao, X. Ouyang, Y. Zhao. "Study on Knowledge Engineering Education Technique,” unpublished.

[6]. H. Steven C, B. H. Dermot, R. Bryan. Relational Frame Theory: A Post-Skinnerian Account of Human Language and Cognition. Now York, Springer Press, 2013.

[7]. P. H. Winston. Artificial Intelligence, New Zealand, Pearson Press, 1992.

[8]. X. Y. Wang, "Study on the Strategy of Structuring Knowledge for High School Chemistry (in Chinese),” Jinan: Shandong Normal University, 2010. 